\title{
Correction to: Widespread colonisation of Tanzanian catchments by introduced Oreochromis tilapia fishes: the legacy from decades of deliberate introduction
}

\author{
Asilatu Shechonge $\cdot$ Benjamin P. Ngatunga $\cdot$ Stephanie J. Bradbeer \\ Julia J. Day · Jennifer J. Freer • Antonia G. P. Ford · Jonathan Kihedu • \\ Tabitha Richmond - Semvua Mzighani - Alan M. Smith • Emmanuel A. Sweke • \\ Rashid Tamatamah $\cdot$ Alexandra M. Tyers $\cdot$ George F. Turner $\cdot$ Martin J. Genner
}

Published online: 30 May 2018

(C) The Author(s) 2018

\section{Correction to: Hydrobiologia}

https://doi.org/10.1007/s10750-018-3597-9

Due to an unfortunate turn of events, four rows in

Table 2 were transposed. Hence, the original article

The original article can be found online at https://doi.org/10.1007/s10750-018-3597-9.

A. Shechonge - B. P. Ngatunga - R. Tamatamah Department of Aquatic Sciences and Fisheries, University of Dar es Salaam, P.O. Box 35064, Dar es Salaam, Tanzania

A. Shechonge $\cdot$ J. Kihedu $\cdot$ S. Mzighani .

E. A. Sweke $\cdot$ R. Tamatamah

Tanzania Fisheries Research Institute (TAFIRI),

P.O. Box 9750, Dar es Salaam, Tanzania

S. J. Bradbeer · J. J. Freer · T. Richmond ·

M. J. Genner $(\varangle)$

School of Biological Sciences, University of Bristol, Life Sciences Building, 24 Tyndall Avenue, Bristol BS8 1TQ, UK

e-mail: m.genner@bristol.ac.uk

\section{J. J. Day}

Department of Genetics, Evolution and Environment, University College London, Darwin Building, Gower

Street, London WC1E 6BT, UK has been corrected. The corrected section (part of 'Minor catchments') of Table 2 is also published here.

A. G. P. Ford - A. M. Tyers - G. F. Turner

School of Biological Sciences, Bangor University,

Bangor, Gwynedd LL57 2UW, UK

\begin{abstract}
A. G. P. Ford
Department of Life Sciences, Centre for Research in Ecology, Whitelands College, University of Roehampton, Holybourne Avenue, London SW15 4JD, UK
\end{abstract}
A. M. Smith
Evolutionary and Environmental Genomics Group, School of Environmental Sciences, University of Hull, Hull HU5 7RX, UK 
Table 2 The number of locations surveyed in catchments across Tanzania, and the number of locations where each species was recorded

\begin{tabular}{|c|c|c|c|c|c|c|c|c|c|c|c|c|c|c|}
\hline \multicolumn{2}{|l|}{$\begin{array}{l}\text { Catchment/ } \\
\text { species }\end{array}$} & \multicolumn{2}{|l|}{$\begin{array}{l}\text { Survey } \\
\text { locations }\end{array}$} & $\begin{array}{l}\text { O. } \\
\text { esculentus }\end{array}$ & $\begin{array}{l}\text { O. } \\
\text { leucostictus }\end{array}$ & \multicolumn{2}{|c|}{$\begin{array}{l}O . \\
\text { niloticus }\end{array}$} & $\begin{array}{l}\text { O. } \\
\text { placidus }\end{array}$ & \multicolumn{2}{|c|}{$\begin{array}{l}O . \\
\text { rukwaensis }\end{array}$} & \multicolumn{2}{|c|}{$\begin{array}{l}O . \\
\text { shiranus }\end{array}$} & $\begin{array}{l}\text { O. } \\
\text { urolepis }\end{array}$ & $\begin{array}{l}\text { O. } \\
\text { jipe }\end{array}$ \\
\hline \multicolumn{15}{|c|}{ Minor catchments } \\
\hline \multicolumn{2}{|l|}{$\begin{array}{l}\text { Dar-es- } \\
\text { Salaam }\end{array}$} & 1 & 0 & & 0 & 1 & & 0 & 0 & & 0 & & 0 & 0 \\
\hline \multicolumn{2}{|l|}{ Lake Kitele } & 1 & 0 & & 0 & 1 & & 0 & 0 & & 0 & & 0 & 0 \\
\hline \multicolumn{2}{|c|}{ Lake Basotu } & 1 & 1 & & 0 & 0 & & 0 & 0 & & 0 & & 0 & 0 \\
\hline \multicolumn{2}{|c|}{ Lake Burungi } & 2 & 0 & & 0 & 2 & & 0 & 0 & & 0 & & 0 & 0 \\
\hline \multicolumn{2}{|l|}{ Lake Chala } & 1 & 0 & & 0 & 0 & & 0 & 0 & & 0 & & 0 & 0 \\
\hline $\begin{array}{l}\text { Catchment/ } \\
\text { species }\end{array}$ & \multicolumn{2}{|c|}{$\begin{array}{l}\text { O. } \\
\text { amphimelas }\end{array}$} & $\begin{array}{l}O . \\
\text { korogw }\end{array}$ & $\begin{array}{l}\text { O. } \\
\text { variabilis }\end{array}$ & \multicolumn{2}{|c|}{$\begin{array}{l}\text { O. } \\
\text { chungruruensis }\end{array}$} & $\begin{array}{l}O . \\
\text { karomo }\end{array}$ & \multicolumn{2}{|c|}{$\begin{array}{l}O . \\
\text { tanganicae }\end{array}$} & \multicolumn{2}{|c|}{$\begin{array}{l}O . \\
\text { malagarasi }\end{array}$} & $\begin{array}{l}\text { O. } \\
\text { hunteri }\end{array}$ & \multicolumn{2}{|c|}{$\begin{array}{l}O \text {. "crater lake } \\
i \text { chambo" }\end{array}$} \\
\hline \multicolumn{15}{|c|}{ Minor catchments } \\
\hline $\begin{array}{l}\text { Dar-es- } \\
\text { Salaam }\end{array}$ & \multicolumn{2}{|l|}{0} & 0 & 0 & 0 & & 0 & 0 & & 0 & & 0 & \multicolumn{2}{|l|}{0} \\
\hline $\begin{array}{l}\text { Lake } \\
\text { Kitele }\end{array}$ & \multicolumn{2}{|l|}{0} & 0 & 0 & 0 & & 0 & 0 & & 0 & & 0 & \multicolumn{2}{|l|}{0} \\
\hline $\begin{array}{l}\text { Lake } \\
\text { Basotu }\end{array}$ & \multicolumn{2}{|l|}{0} & 0 & 0 & 0 & & 0 & 0 & & 0 & & 0 & \multicolumn{2}{|l|}{0} \\
\hline $\begin{array}{l}\text { Lake } \\
\text { Burungi }\end{array}$ & \multicolumn{2}{|l|}{0} & 0 & 0 & 0 & & 0 & 0 & & 0 & & 0 & \multicolumn{2}{|l|}{0} \\
\hline $\begin{array}{l}\text { Lake } \\
\text { Chala }\end{array}$ & \multicolumn{2}{|l|}{0} & 0 & 0 & \multicolumn{2}{|l|}{0} & 0 & 0 & \multicolumn{2}{|r|}{0} & & 1 & 0 & \\
\hline
\end{tabular}

